\title{
La gestión del conocimiento, una política organizacional para la empresa de hoy
}

\author{
Knowledge management, organizational policy for business today \\ Erica Janet Agudelo Ceballos ${ }^{1} \quad$ Alejandro Valencia Arias ${ }^{1 *}$ \\ Recibido 26 de mayo de 2017, aceptado 12 de enero de 2018 \\ Received: May 26, 2017 Accepted: January 12, 2018
}

\begin{abstract}
RESUMEN
El presente artículo, es producto del acercamiento teórico-práctico a la Gestión del Conocimiento, modelo gerencial que llevado a cabo en la organización como estrategia, posibilita no solo el direccionamiento de la misma, sino también el entendimiento de su realidad frente al entorno en el que se desenvuelve. Inicialmente, se despliegan algunos conceptos teóricos del modelo gerencial, con el fin de definir sus características y elementos más representativos. Posteriormente, se establece una relación de análisis mediante un estudio de caso con el fin de identificar la pertinencia de un modelo de Gestión del Conocimiento para la actividad organizacional. De esta manera, se concluye que éste debe ser parte de la política de dirección que se adopte en la empresa de hoy; pues el aprendizaje organizativo, abre la compuerta al liderazgo, de hecho, al consolidarlo como directriz institucional, el líder proporciona los recursos necesarios para que el conocimiento se formule, se implante y se sostenga como una estrategia de largo plazo.
\end{abstract}

Palabras clave: Gestión del conocimiento, modelo gerencial, sociedad del conocimiento, organización.

\begin{abstract}
This article is the product of the theoretical and practical approach to knowledge management, management model implemented in the organization as a strategy allows not only addressing the same but the understanding of the reality facing the environment in which operates. Initially, some theoretical concepts of management model deployed, to define its characteristics and most representative elements. Subsequently, a ratio analysis using a case study to identify the relevance of a knowledge management model for organizational activity, which allow conclusions and finally concluded that this model should be part of the set management policy to be adopted in the company today; as organizational learning, leadership opens the gate, in fact, to strengthen knowledge management as a policy, the leader provides the resources for knowledge to be made, implanted and sustained as a long-term strategy.
\end{abstract}

Keywords: Knowledge management, knowledge society, organization.

\section{INTRODUCCIÓN}

Las sociedades han evolucionado de acuerdo con los factores estratégicos de desarrollo que para cada momento histórico se han determinado. En la primera Revolución Industrial, por ejemplo, fue la máquina, y en la segunda, la Tecnología. Dichos cambios, se han dado ante la imperante necesidad de transformaciones que el hombre mismo ha generado, especialmente por su relación con el entorno. Actualmente, en pleno siglo XXI, asistimos a la Sociedad del Conocimiento [1-2], período en

\footnotetext{
1 Departamento en Ciencias Administrativas, Instituto Tecnológico Metropolitano. Dirección: Cl. 54a \#30-99. Medellín, Colombia. Email: ericaagudelo@gmail.com; jhoanyvalencia@itm.edu.co

* Autor de Correspondencia
} 
el cual se observa que la capacidad de aprendizaje es la puerta de entrada a un desarrollo sostenible e integral. Para Drucker [3] "Ha sobrevenido un cambio súbito y radical en el significado y los efectos del saber para la sociedad. Porque ahora podemos organizar a individuos de alta pericia y sabiduría para el trabajo colectivo mediante el ejercicio del juicio responsable, el individuo altamente educado se ha convertido en el recurso central de la sociedad de hoy". De forma, que la capacidad del trabajo productivo, como bien lo afirmó, se basará en la mente y no en la mano.

A partir del siglo XIX, se ha introducido el concepto Sociedad del Conocimiento gracias a la discusión que se ha dado en torno al valor, entre ellos, el económico [4], de la educación y su aporte al desarrollo de la sociedad y en particular, del sujeto al tornarlo más cualificado [5]. Bien lo decía Drucker [6]: “...en la nueva economía, el conocimiento es el principal recurso para los individuos y la economía. Tierra, trabajo y capital no desaparecerán pero serán secundarios...", esto significa que el conocimiento toma mayor relevancia en comparación con los demás factores de producción tradicionales, debido a las nuevas condiciones ambientales que afectan a las empresas [7]. De tal forma, que puede afirmarse que el conocimiento dinamiza el mundo, y por lo tanto, todo aquello que lo compone está llamado a gestionarlo. Es decir, los individuos y las organizaciones, actores sociales, están convocados a darle un valor, apropiarse de él y darle un uso, paso final del proceso de la gestión del conocimiento [8].

Para observar el significado y el papel de la Gestión del Conocimiento en torno a la organización, se despliegan a continuación algunos conceptos teóricos y se da paso a su aplicación en un análisis de caso. Finalmente, se presentan las principales conclusiones a las que se llega de manera general sobre este ejercicio.

\section{LA GESTIÓN DEL CONOCIMIENTO}

Como ya se ha expresado, el Conocimiento juega un papel relevante en la sociedad [9], si bien, su rol determina en gran medida la capacidad de acción tanto de individuos como de organizaciones, el conocimiento por sí solo no tiene implicancia, pero en manos de "alguien" que lo gestione jugará un papel más importante y por lo tanto, protagónico en donde se lleve a cabo [9].
En el caso concreto de la organización, el conocimiento se ha convertido, y para quienes así lo consideran, en otro activo más [10-11]; pero no uno cualquiera, sino aquel que le permite consolidar un proyecto de más largo alcance, en otras palabras, hacerla más competitiva en un entorno dinámico [12]. Pero una organización competitiva, es también aquella que piensa en el grado de conocimiento de su recurso humano, y éste, dado en sus niveles de formación y experiencia. De ahí, que Castells [13] precise del concepto como un aspecto "fundamental en todas las sociedades (...) el atributo de una forma específica de organización social en la que la generación, el procesamiento y la transmisión de la información se convierten en las fuentes fundamentales de la productividad y el poder".

Pero, ¿Qué es Gestión del Conocimiento?, para Gottschalk [14] es el "método para simplificar y mejorar el proceso de compartir, distribuir, crear, capturar y entender el conocimiento dentro de una compañía." En otras palabras, es la relación empleadoempresa orientada a gestionar la información; es decir, identificarla, seleccionarla, organizarla y darle un uso. Así, el conocimiento es la posibilidad de entender la realidad de la organización mediante la cooperación de quienes en ella intervienen y, gracias al aprendizaje se pueden tomar y fortalecer las decisiones [15]. Para la empresa de hoy, inscrita en un entorno globalizado [16] y menos complejo gracias a la desconfiguración de barreras, el conocimiento es su llamado a la acción y al mejoramiento continuo.

\section{Embudo del Conocimiento}

La organización que se piensa, asume que la información circulante atraviesa un proceso llamado el Embudo del Conocimiento [17] y que se observa en la Figura 1:

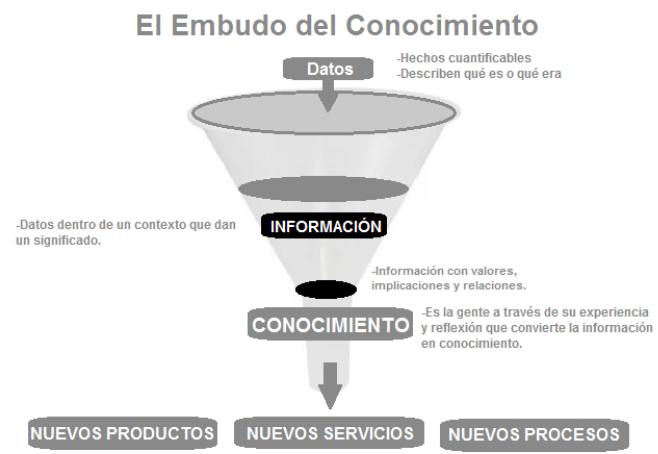

Figura 1. Embudo del Conocimiento. Fundación Iberoamericana del Conocimiento [17] 
La representación del embudo, permite establecer que los datos son un registro que por sí solo no tienen propósito, de manera que no tienen descripción alguna para la toma de decisiones; la información, corresponde a los datos transformados mediante la asignación de un significado que se le da cuando entra en contacto con algún contexto, de manera que adquiere una propiedad; por su parte, el conocimiento, es la interrelación entre datos e información que se transforman en conocimiento, y son las personas quienes la cargan, según el contexto, con su experiencia y vivencias.

\section{Tipos de Conocimiento}

Nonaka y Takeuchi [18] exponen las ideas que sustentan la teoría -que lleva por nombre el mismo de su obrapor medio de la cual establecen un modelo para la generación del conocimiento, el cual se da mediante la interacción de uno explícito (formal y sistemático, fácilmente comunicado y compartido) y otro tácito (de carácter personal, como modelos mentales, creencias, experiencias, perspectivas individuales; difícil comunicarlo a los demás). En la Figura 2, se diagrama el proceso de interacción que funciona en cuatro formas de "Espiral del Conocimiento":

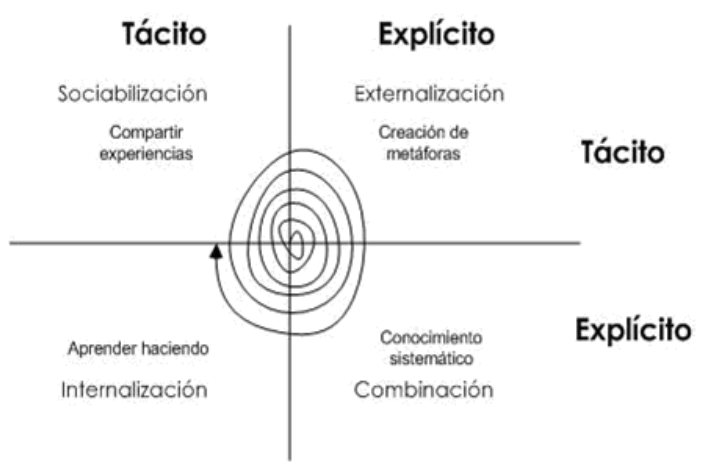

Figura 2. Proceso de creación del conocimiento de Nonaka y Takeuchi [1].

Como se observa, la imagen representa la interacción dinámica mediante la cual se establecen las formas de: socialización (lograr conocimiento tácito compartiendo experiencias gracias a la oralidad, el material escrito y las tradiciones); externalización (cambiar conocimiento tácito por explícito a través del empleo de metáforas que se integran a la cultura de la organización); combinación, (instaurar conocimiento explícito al agrupar conocimiento explícito derivado de diálogos, reuniones, correos, entre otros); y, finalmente, internalización, (incorporar conocimiento explícito en conocimiento tácito, que al incorporarse en los individuos de la organización se constituye en modelos mentales o experiencias de trabajo).

\section{Proceso de la Gestión del Conocimiento}

Toda organización, se propone la definición de un ciclo por medio del cual consolidar el proceso para gestionar el conocimiento, de modo que éste se articule con la estrategia y sea el resultado de una construcción conjunta de todas las áreas [19]. A continuación, se presenta en la Figura 3 un modelo del proceso:

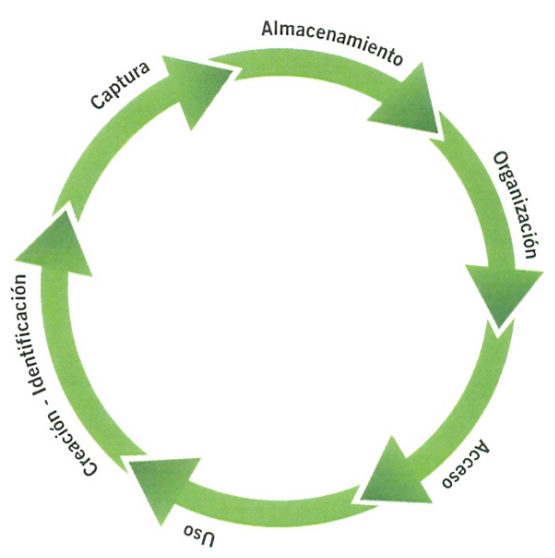

Figura 3. Proceso de la Gestión del Conocimiento [20].

En el ciclo, siguiendo a Nonaka y Takeuchi [18], se plantea la idea de cómo hacer para que ese conocimiento se transfiera a la organización, de forma que la creación-identificación es la detección del conocimiento para poder gestionarlo; la captura se refiere a la generación de un ambiente para que se dé el intercambio de experiencias; el almacenamiento es entendido como algo dinámico; la organización como el proceso estructurado en un mismo lenguaje; el acceso es la posibilidad que todas las personas de la organización accedan a él; y, por último, el uso está determinado por la utilidad que cada cual le dé según su necesidad.

La organización debe, para aplicar este proceso, precisar con claridad las responsabilidades que se asumen por parte de cada uno de sus funcionarios, si bien todos gestionan el conocimiento, todos igualmente deben asumir un rol al entregar y recibir 
información que se transforma en conocimiento como ya se dijo; adicionalmente, porque el proceso se perfecciona en la medida que su dinamismo le regenera.

\section{Elementos de la Gestión del Conocimiento}

La Gestión del Conocimiento para ser desarrollado, no demanda simplemente la identificación del conocimiento para su procesamiento y la creencia de su importancia para el establecimiento de una ventaja competitiva para que sea sostenible, requiere la observación clara de unos elementos que le posibiliten su inserción al interior de la organización. Bueno [21], presentó la Triada Conceptual en la que se relacionan los elementos de la sociedad del conocimiento bajo un enfoque holístico; es decir, integrador. En la Figura 4, se precisan dichos elementos que le aportan, como activos intangibles, valor al conocimiento que capitaliza a la organización que la gestiona:

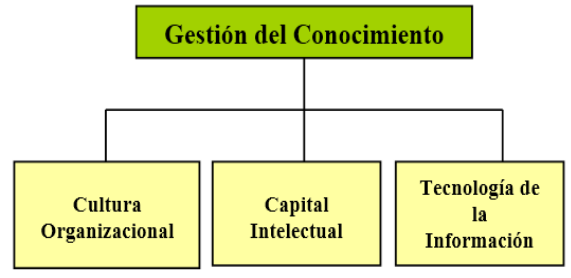

Figura 4. Elementos de la Gestión del Conocimiento [21].

De acuerdo con el autor, el concepto para cada elemento se detalla así: la Cultura Organizacional es el reflejo de la visión compartida por todos los empleados de la organización y los resultados de su aplicación; el Capital Intelectual, es el total de activos intangibles de la empresa y que generan un alto valor agregado, algunos son: habilidades, destrezas, sentimientos, creencias; y, la Tecnología de la Información, que hace referencia al conjunto de herramientas informáticas y de telecomunicaciones que facilitan la ejecución de determinadas actividades.

Con la interrelación de estos tres elementos intangibles, la organización actúa bajo el entendimiento que también pueden ser gestionados estratégicamente ya que componen aquello que en la actualidad se conoce como el Know-how o el saber-cómo y que está directamente relacionado. Según Nonaka y
Takeuchi [18], con las experiencias que va dejando el proceso de la gestión y el establecimiento de una cultura compartida; es decir, una dimensión técnica en cada individuo (cómo realizar una tarea específica).

\section{El aprendizaje organizativo}

Senge [22], desarrolló en su obra "La Quinta Disciplina" una propuesta respecto al proceso de aprendizaje de las organizaciones, así como una mirada a las barreras que imposibilitan a la organización inteligente. Para el efecto, planteó cinco disciplinas que se entienden como la forma de poder construir el aprendizaje organizativo; de llevarse a cabo, serán empresas que se desarrollarán de manera exitosa ya que asumirán el aprendizaje como la constante de su proceso organizativo y por ende, la adoptarán como parte de sus políticas estratégicas y, a su vez, como valor de capital intangible. A continuación, en la Tabla 1 se relacionan las disciplinas de Senge [22]:

Tabla 1. Disciplinas de Senge

\begin{tabular}{c|c}
\hline Disciplina & Síntesis \\
\hline $\begin{array}{c}\text { Dominio } \\
\text { Personal }\end{array}$ & $\begin{array}{c}\text { Una organización aprende cuando sus } \\
\text { individuos también procuran hacerlo. }\end{array}$ \\
\hline $\begin{array}{c}\text { Modelos } \\
\text { Mentales }\end{array}$ & $\begin{array}{c}\text { La estructura prenocional del individuo debe } \\
\text { abrirse para percibir las nuevas ideas y así } \\
\text { practicarlas. }\end{array}$ \\
\hline $\begin{array}{c}\text { Visión } \\
\text { Compartida }\end{array}$ & $\begin{array}{c}\text { Una visión compartida por todos los } \\
\text { miembros de la organización, es una muestra } \\
\text { clara de direccionalidad en su estructura. }\end{array}$ \\
\hline $\begin{array}{c}\text { Aprendizaje en } \\
\text { Equipo }\end{array}$ & $\begin{array}{c}\text { El trabajo en equipo produce siempre más y } \\
\text { mejores resultados en el esfuerzo individual. }\end{array}$ \\
\hline $\begin{array}{c}\text { Pensamiento } \\
\text { Sistémico }\end{array}$ & $\begin{array}{c}\text { La realidad vista en su conjunto, es la forma } \\
\text { en que las organizaciones inteligentes miran } \\
\text { objetivamente su contexto. }\end{array}$ \\
\hline
\end{tabular}

El concepto de pensamiento sistémico como la acción integral, es decir, el resultado gracias a la integración de todas las unidades, es la disciplina que según Senge [22] constituye a las anteriores; así, una organización que las adopte como parte de sus políticas, actuará bajo el principio de la sinergia donde el resultado que se expone se da mediante la maximización de la unión de dos o más personas.

De otro lado, de acuerdo con el mismo autor y valiéndose de la parábola de la rana hervida para establecer una analogía respecto al comportamiento adaptativo, precisa que las organizaciones deben evaluar sus procesos de aprendizaje de manera tal, que puedan establecer e implantar, oportunamente, a modo de políticas, los resultados que esta arroje, 
ya sean: correctivos para la mejora continua, establecimiento de las buenas prácticas, optimización de tiempos y recursos, entre otros.

\section{FACTORES CLAVE EN LA GESTIÓN DEL CONOCIMIENTO}

Toda organización, sin importar su actividad económica, está convocada a poner en marcha y fortalecer la implementación formal del conocimiento como parte de sus procesos de gestión [22], y aunque esté expuesta a múltiples desafíos [23], el conocimiento se refiere a la posibilidad estratégica con que cuenta cada una para actuar en su entorno según su capacidad para administrarlo.

Los modelos gerenciales están construidos sobre la base de modelos mentales que se arraigan al interior de la organización [24] y que, por tanto, demandan, como conceptúan algunos sociólogos y psicólogos, saltar el obstáculo del "prisionero de la cultura" [21]. Es decir, no se pueden perder de vista los objetivos que fundamentan la Gestión del Conocimiento y para ello, es necesario contar con factores clave que rompan el esquema de que todo está bien y por tanto, también lo que se hace, cómo se hace y quién lo hace. Entre los factores citados por Senge [21], se destacan:

Factor de Liderazgo: entendido desde un proceso gerencial, es la persona que guía, dinamiza, orienta y conduce a sus seguidores e influye positivamente en ellos. La clave del liderazgo se encuentra en las fortalezas del equipo y las relaciones que se basan en la confianza y el respeto mutuo en cuanto a los aportes y sugerencias. El liderazgo participativo, como factor clave para la Gestión del Conocimiento, propende porque las acciones del líder potencien la generación del conocimiento ya que despierta el interés de los individuos por un trabajo propositivo y alineado con los objetivos de la organización.

Factor de Trabajo en Equipo: el trabajo en equipo se emprende gracias a la visión compartida y al logro de objetivos comunes, de allí, que la clave para el éxito sea el bien común y nunca el trabajo individual. En este sentido, implica que el grupo de personas trabajen de manera coordinada y cooperen para la obtención del mismo resultado. Este factor clave para la Gestión del Conocimiento, saca a flote las habilidades de los individuos y de la organización, de modo que se promueva el sentido de pertenencia y un mayor nivel de desempeño.

Factor de Alto Rendimiento: el trabajo colaborativo pone en juego la complejidad de competencias y responsabilidades, es el factor diferencial del trabajo en equipo. Cada participante aporta su conocimiento y por tanto, a la estrategia como herramienta de trabajo. De esta manera, el trabajo de un equipo de alto rendimiento implica, además de cohesión, que las tareas y los objetivos se alcancen en términos de eficacia y eficiencia. Por lo tanto, este factor clave para la Gestión del Conocimiento, propone círculos de confianza en los que la comunicación es imperativa para obtener resultados funcionales.

Adicionalmente, uno de los factores que va ganando mayor protagonismo y se vuelve transversal a las organizaciones, es la incorporación de Tecnologías de Información y Comunicación (TIC) como herramienta de soporte a la gestión del conocimiento [25], los cuales se han acompañado de estrategias como los modelos de adopción tecnológica [26] que se han acompañado de herramientas sistémicas como el modelado mediante agentes [27], la creación de sistemas multiagentes [28] y la implementación de comunidades de aprendizaje virtuales [29] para mejorar el respaldo y acceso a la información y el proceso de transferencia de tecnologías emergentes [30].

\section{APLICACIÓN: ESTUDIO DE CASO.}

Un acercamiento a la aplicación del modelo Gestión del Conocimiento, es la propuesta que a continuación se desarrolla mediante el Estudio de Caso Investigativo; según Hartley [31] "el estudio de casos consiste en un estudio detallado, a menudo durante un periodo de tiempo, de un fenómeno en el contexto en que ocurre". De tal forma, que el objetivo es comprender la peculiaridad del caso, la forma cómo funciona y las relaciones que se presentan; es comprender "el significado de una experiencia", ya que el producto final puede ser una descripción de tipo cualitativo [26]. En concordancia, el estudio de caso se aborda a partir de la comparación entre la teoría previamente expuesta y el ejercicio práctico al interior de la empresa seleccionada; el desarrollo se llevó a cabo de acuerdo con las fases de León y Montero [32]: $1^{\circ}$ La selección y definición del caso, $2^{\circ}$ Elaboración de una lista de preguntas, $3^{\circ}$ Localización de las fuentes de datos, $4^{\circ}$ Análisis e interpretación, y $5^{\circ}$ Elaboración del 
informe. Para avanzar en el estudio del caso, se planteó la pregunta ¿Cómo se da el proceso de la Gestión del Conocimiento en la organización?; a ésta se dará respuesta en la medida que se presentan los avances del ejercicio.

La empresa objeto del caso es la Asociación de Profesores de la Universidad Nacional de Colombia -APUN Medellín-, entidad sin ánimo de lucro fundada hace 50 años por profesores de la institución universitaria, interesados en agremiarse inicialmente para defender y proteger sus derechos como estamento docente. Paulatinamente, fue implementando servicios de bienestar para sus asociados, entre ellos el crédito y las alianzas con proveedores para procurar bienes y servicios; al año 2014, contaba con 460 profesores asociados. Es, igualmente, promotora de eventos académicos y comerciales al interior del campus universitario.

A nivel interno, entre sus competidores directos se cuentan la Cooperativa de Servicios -COOSERVUNAL- y el Fondo de Empleados Docentes -FODUN-, y está afiliada a la Federación Nacional de Profesores Universitarios -FENALPROU-; por relación gremial, se vincula a la actividad de otros grupos, entre ellos la Asociación Sindical de Profesores Universitarios -ASPU-, y la Asociación de Profesores de la Universidad de Antioquia-ASOPRUDEA-, entre otros.

Entre las condiciones iniciales para el análisis, se observó lo siguiente:

- El talento humano de la empresa es cualificado dada la formación académica y el nivel de experiencia, reflejado en las áreas de desempeño.

- Los empleados vinculados tienen una antigüedad promedio de entre cuatro y veinte años de servicio, lo que representa un alto conocimiento interno y externo de la institución, y un conocimiento implícito y explícito acumulado.

- Las actividades propias de la organización dan cuenta de un ejercicio de aplicación del conocimiento, asimismo la experiencia que éstos arrojan, son parte del capital acumulado de su aprendizaje organizativo.

- La empresa no tiene formalizado a través de protocolos o manuales los procesos de gestión. Estos se dan de manera implícita.

\section{TÉCNICA DE OBSERVACIÓN}

Esta técnica de investigación se llevó a cabo mediante el análisis de la siguiente información suministrada por la empresa:

Respecto a la base social de la APUN Medellín, la distribución es la siguiente: Jubilados un total de 216, equivalente a 24\%; los Docentes un total de 239, equivalente a 26,50\%; Otros usuarios (empleados de la Universidad Nacional de Colombia Sede Medellín) un total de 446, equivalente a 49,50\%; para total un general de 901 usuarios.

De acuerdo con el Informe de Actividades a 2014, se registra en actividades por año, que contempla ferias, muestras comerciales, foros, conferencias, conversatorios, entre otros; el balance arroja el siguiente comparativo: en la vigencia 2011, un total de 11 actividades; a 2012, 17 actividades; a 2013, 26 en total; y, a 2014, un total de 31.

El registro en la página web, reportó en el balance a 2014 sobre las actividades correspondientes a noticias, documentos y boletines; éstos registraron: noticias a 2013, 72 y a 2014, 98; documentos a 2013 , 53 y a 2014, 61; y boletines a 2013, 16 y a 2014, 22 . Acorde con la información anterior, se colige:

- El movimiento de la base social se da gracias a la gestión de cada una de las áreas; para que se lleven a cabo, demandan la existencia de una gestión por procesos donde los objetivos misionales y la visión compartida son fundamentales.

- Ante la propuesta de nuevos procesos o nuevas tareas, todas las áreas reciben la idea y deben aportar en la construcción y puesta en marcha.

- El balance de actividades da cuenta que el conocimiento es la base de la gestión organizacional y por tanto, de su aprendizaje organizativo en relación con sus entornos.

- Los registros web, señalan que la empresa gestiona el conocimiento mediante el uso y aplicación de las herramientas tecnológicas. De esta manera, la información se transforma en conocimiento cuando al comunicársela a los receptores, éstos dan respuesta mediante la decodificación, a modo de retroalimentación.

- La retroalimentación entre las áreas de la empresa, implica compartir las experiencias 
que se dan durante el ejercicio laboral a modo de aprendizaje.

- Se intercambia la información por diversas fuentes, se contextualiza y categoriza de manera que se transforme en conocimiento para la organización.

- Se incorporan los resultados de las actividades a la cultura de la empresa y se ponen en práctica generando así un nuevo aprendizaje.

- La retroalimentación entre las áreas de trabajo, implica compartir las experiencias que se dan durante el ejercicio laboral y así se intercambia la información por diversas fuentes, lo cual es un proceso clave de gestión del conocimiento para las organizaciones [33].

- Al ponerse en práctica e incorporar los resultados a la cultura de la Asociación, se genera un nuevo aprendizaje y por ende, una inserción al modelo mental de los individuos.

- Se observa que la visión y misión de la empresa, son compartidas por el talento humano, así como los valores, ideas y conductas, lo que representa la conformación de la cultura organizacional, elemento para la gestión del conocimiento.

\section{TÉCNICA DE ENCUESTA}

Con el fin de contrastar la información anterior, e identificar cómo se aplican los procesos de la Gestión del Conocimiento al interior de la empresa, se llevó a cabo esta técnica con ocho empleados, distribuidos entre el mando alto y medio de la Asociación. El formato de encuesta seleccionado, es tomado de Bernal [34].

Los criterios para su aplicación, fueron los establecidos por el autor:

“(...) 12 enunciados (ítems) relacionados con la apropiación, aplicación y generación de conocimiento en las organizaciones. Las preguntas fueron concebidas en cuanto tienen relación con la capacitación de las personas que laboran en la empresa, su análisis del entorno y el uso de las TIC. (...) fue estructurado bajo el criterio de la escala Likert, donde el encuestado califica cada ítem, según su percepción, en puntuaciones de 1 a 4; donde $1=$ Totalmente en desacuerdo, $2=$ Parcialmente en desacuerdo, $3=$ =Parcialmente de acuerdo y 4=Totalmente de acuerdo."
A continuación, la Tabla 2 presenta los resultados del ejercicio de análisis comenzando con el porcentaje de mayor representatividad:

Tabla 2. Modelo de encuesta y resultados sobre aplicación de Gestión del Conocimiento en APUN Medellín.

\begin{tabular}{lc}
\hline \multicolumn{1}{c}{ Enunciado de la pregunta } & Puntuación \\
\hline $\begin{array}{l}\text { En la organización todos estamos informados } \\
\text { de los resultados de ésta. }\end{array}$ & $95 \%$ \\
\hline $\begin{array}{l}\text { En esta organización siempre se aprovecha el } \\
\text { conocimiento que tienen las personas. }\end{array}$ & $94 \%$ \\
\hline $\begin{array}{l}\text { La organización aprende de la interacción con } \\
\text { el entorno. }\end{array}$ & $91 \%$ \\
\hline $\begin{array}{l}\text { En la organización se usan de forma permanente } \\
\text { las TIC. }\end{array}$ & $91 \%$ \\
\hline $\begin{array}{l}\text { En la organización solemos estar atentos a los } \\
\text { cambios del entorno. }\end{array}$ & $88 \%$ \\
\hline $\begin{array}{l}\text { En la organización se suele incorporar nuevos } \\
\text { conocimientos a la actividad productiva. }\end{array}$ & $85 \%$ \\
\hline $\begin{array}{l}\text { Nuestra organización siempre se esfuerza porque } \\
\text { aprendamos unos de otros. }\end{array}$ & $84 \%$ \\
\hline $\begin{array}{l}\text { En la organización se reconoce y estimula la } \\
\text { creatividad y la innovación. }\end{array}$ & $81 \%$ \\
\hline $\begin{array}{l}\text { En la organización trabajamos pensando en los } \\
\text { cambios del futuro. }\end{array}$ & $80 \%$ \\
\hline $\begin{array}{l}\text { En la organización siempre todos nos actualizamos } \\
\text { de forma permanente. }\end{array}$ & $78 \%$ \\
\hline $\begin{array}{l}\text { En esta organización siempre es prioritaria la } \\
\text { capacitación de las personas. }\end{array}$ & $70 \%$ \\
\hline $\begin{array}{l}\text { En la organización se suelen utilizar resultados } \\
\text { de investigación (internas y externas). }\end{array}$ & \\
\hline
\end{tabular}

Tanto el ejercicio de observación como la encuesta, permiten conjuntamente colegir que:

- Los resultados del ejercicio anterior, reportan información útil para la toma de decisiones en la organización.

- El proceso de compartir la información es una actividad plenamente establecida como una dinámica para la generación de conocimiento.

- Ante la creación de nuevos procesos, gracias a la retroalimentación por áreas, se generan nuevos aprendizajes que capitalizan el valor del conocimiento y adquiere sentido para los empleados.

- Al darse la socialización, el conocimiento explícito y la interiorización, elementos del Proceso de creación del Conocimiento [35], la empresa fortalece la generación del conocimiento como una constante (espiral) en las actividades diarias.

- El componente del Capital Intelectual [36], la Cultura Organizacional [37] y la Tecnología de la Información [38], elementos de la Gerencia 
del Conocimiento, son aspectos que APUN Medellín no inserta en su política organizacional ya que no dimensiona con suficiente claridad que son patrones que posibilitan su proyección como una entidad competitiva en el entorno.

- La Asociación de Profesores no reconoce de manera formal que al interior de la organización se lleva a cabo este Modelo Gerencial; por lo tanto, permitiendo confirmar que lo hace de manera explícita, en cuyo caso, la propuesta es formalizarlo como parte de sus procesos y su cultura.

\section{DISCUSIÓN}

La gestión del conocimiento ha sido definida como la estrategia a través de la cual las organizaciones identifican el potencial intelectual de su talento humano, con la finalidad de encaminarlo al cumplimiento de los objetivos estratégicos del negocio [39], en este sentido se observa que los encuestados perciben en un $94 \%$ que la organización aprovecha el conocimiento que tienen las personas y en un $91 \%$ que la organización aprende de la interacción con el entorno, lo que en cierta medida respalda el potencial del personal y la articulación de este con la gestión del conocimiento.

Adicionalmente, es importante mencionar que la transformación de las habilidades corporativas y el conocimiento implícito en cada uno de sus funcionarios y en los procesos en general, se haya constituido en el capital intelectual corporativo, pasando de factores tangibles a intangibles, tal como lo sugiere Monagas-Docasal [40] y esto se refleja en que el $85 \%$ de los encuestados plantea que en la organización se suele incorporar nuevos conocimientos a la actividad productiva.

En la literatura se encuentran estudios afines a los hallazgos de este manuscrito, como es el caso del desarrollado por Acosta quien construyó un modelo explicativo que correlaciona la gestión del conocimiento y las habilidades innovadoras, respecto a su impacto en las dinámicas empresariales [41], lo cual se hace tangible en este estudio ya que el $81 \%$ de los encuestados plantea que en la organización se reconoce y estimula la creatividad y la innovación.

Consecuentemente, Cárcel y Roldán develan en su investigación la importancia de identificar las barreras para la gestión del conocimiento, en organizaciones de tipo industrial, particularmente en lo que se refiere a los profesionales que poseen un alto grado de conocimiento tácito [42].

Sin embargo, aunque la gestión del conocimiento cobra cada vez mayor fuerza en el ámbito empresarial, autores como Arboleda coinciden con algunos de los resultados expuestos por esta investigación, al referir el desconocimiento que aún persiste sobre la temática en el personal directivo de las organizaciones, y por ende la ambigüedad a la cual esta situación da lugar en el momento de incursionar en el diseño de estrategias para capitalizar el conocimiento existente en las empresas [43]. Entre estas estrategias, se sugiere para la organización la incorporación de permitan utilizar resultados de investigación (internas como externas) en beneficio de la mejora continua de los procesos organizacionales, ya que este es el aspecto con puntuación más baja en todo el estudio de caso.

Adicionalmente, cabe destacar que la implementación de estrategias afines a la gestión del conocimiento pueden ser variables entre un tipo de organización y otra, lo cual obedece a su contexto. Romo, Villalobos y Guadalupe, en su estudio sobre la forma cómo las instituciones de educación superior llevan a la praxis este importante tema de actualidad, exponen la dificultad implícita del proceso por la diversidad de factores que intervienen, como es el tipo de formación de los docentes, sus habilidades, el conocimiento tácito y el relacionamiento que tienen con su entorno [44].

Precisamente, en el caso de la Asociación de Profesores, entidad gremial docente, según se colige del ejercicio metodológico aplicado, el conocimiento explícito hace parte del hacer y el conocimiento implícito del ser; mediante esta combinación, la empresa logra sus tareas, es decir, ser "una organización especializada", como Drucker afirmó. En otras palabras, la APUN Medellín, gestiona el conocimiento mediante la circulación de la información, eso sí, aprovechando el aprendizaje acumulado como capital organizacional y generando nuevo conocimiento a partir de la interacción y la acción entre los públicos internos y su relacionamiento con el entorno externo [45-46].

\section{CONCLUSIONES}

El modelo Gestión del Conocimiento es una herramienta para la organización que bien podría ser implementado como estrategia que posibilite, tanto 
su direccionamiento como su entendimiento de la realidad a la que asiste respecto al entorno en el que se inserta. Al precisar que las sociedades evolucionan, se está afirmando que los cambios son la alternativa para una mejor forma de entender lo que se hace o, por lo menos, hacerlo mejor así se esté haciendo. De esta manera, en el marco de la globalización y ante una sociedad del conocimiento, la educación adquiere la dimensión de capital intangible; su valor está evidenciado en los resultados que logran, por ejemplo, los individuos y las organizaciones por sí mismos, así como el resultado que arroja si se diera una interrelación mediada por la vida laboral.

Si el conocimiento dinamiza el mundo, es válido considerar que el conocimiento integrado a la organización dinamiza su valor al convertirla en una ventaja competitiva; y para ello, gestionar dicho conocimiento es la manera de hacerlo productivo $\mathrm{y}$ transformarlo en una herramienta que hace parte de la práctica explícita e implícita en la vida de la organización. Tal práctica, se logra si la organización, en su proceso de asimilación, comprende que es necesario formalizarla mediante políticas estratégicas. Es decir, hacerla parte de la vida organizacional que no es otra cosa que la cultura.

Cuando las personas comprenden con suficiente claridad que una acción que pudiera parecer tan simple como establecer un contacto a través de la intranet, la creación de una base de datos, las relaciones con los stakeholders, entre otras, resultan ser parte de un proceso de aprendizaje organizativo, dan valor a la información y la contextualizan porque saben que pasará a ser conocimiento -elemento diferenciador respecto a un competidor-, y se dará un acto de interiorización, lo cual permitiría a la organización adoptar nuevas estrategias que den valor agregado a sus procesos organizacionales.

Por su parte, el aprendizaje organizativo abre la compuerta al liderazgo, de hecho, al consolidar la Gestión del Conocimiento como una política, el líder proporciona los recursos para que el conocimiento se formule, se implante y se sostenga como una estrategia de largo plazo. Una razón más para que la Gestión del Conocimiento se constituya a través de políticas que se desarrollen a través de procesos y procedimientos que lo formalicen y sean de estricto cumplimiento. Para lograrlo, dichas políticas deben comunicarse y así volvemos al hecho que la cultura organizacional es el puente.

Un valor sostenible en el tiempo es el capital humano, precisamente el conocimiento se concreta en la mente de un individuo porque allí es donde se aloja y ahí está el punto de partida para gestionarlo. Las personas crean la cultura de la que aquí se ha hablado, y ella tiene que articularse con el aprendizaje que genera el sentir, el pensar y el actuar de los individuos, así como de las relaciones interpersonales que se gestan al interior de la organización. El resultado que arroja la sumatoria de estos aspectos, intervendría del mismo modo en el planteamiento de políticas que procuren la Gestión del Conocimiento.

En la sociedad actual, la denominada ventaja competitiva no se sustrae del capital intangible, es decir, del conocimiento; por el contrario, está fuertemente vinculada como pudo corroborarse mediante el sustento teórico abordado en el presente estudio. Toda práctica organizacional, está mediada por el capital humano e intelectual, justamente, de estos es que se materializa la innovación como fuente de crecimiento y sostenibilidad organizacional, en medio de entornos cambiantes y cada vez más turbulentos, en otras palabras, globalizados. Hoy más que antes, las organizaciones deben capturar el conocimiento como el activo más duradero y sostenible en el tiempo, incluso, más que la mano de obra como factor de producción; en la actualidad, la transversalidad en la gestión organizacional está dada por el conocimiento y el nivel de especialidad de los individuos quienes son realmente quienes disponen de él.

Es, ante este contexto, que la organización objeto de estudio de la aplicación teórico-práctica, permite establecer, como característica general de las organizaciones, que las políticas que se planteen, derivadas de la Gestión del Conocimiento como estrategia para la acción, trazará el rumbo y los actores que intervendrán en su adopción; asimismo, establecerá si dichas políticas serán descriptivas o prescriptivas (que ordenan o son determinantes). Finalmente, y a partir de lo evidenciado en el estudio, se colige que es la alta dirección de la organización quien deberá tener en cuenta al estructurar y formalizar tales políticas, que todo resultado derivado de la Gestión del Conocimiento tendrá que servir como guía para la toma de decisiones 
ya que este también es el sentido del modelo. Si bien, toda organización estructura sus modelos de gestión, es ante la Gestión del Conocimiento que se identifica el cómo y el porqué del capital humano y del capital intelectual como factores clave del desempeño especializado y cada vez más centrado en el aprendizaje organizativo, elemento diferenciador y de valor de las organizaciones competentes en la era del conocimiento.

Como trabajo futuro se recomienda el uso de taxonomías de conocimiento [47] sirve como una guía inicial de trabajo para los ingenieros de conocimiento, ya que se puede hacer una selección mucho más acertada de las técnicas a emplear en los procesos representación de conocimientos. Adicionalmente, se recomienda plantear trabajos de gestión de conocimiento con modelos que respondan a las necesidades y estrategias particulares de las PYME [48] dadas sus particularidades económicas y organizacionales.

\section{REFERENCIAS}

[1] A. Atehortúa, J. Valencia y R. Bustamante. "Gestión del Conocimiento Organizacional, un enfoque práctico". ICONTEC. 1. Bogotá, Colombia. 2011. ISBN: 9789584484178.

[2] N. Sedziuviene and J. Vveinhardt. "Competitiveness and innovations: Role of knowledge management at a knowledge organization". Engineering Economics. Vol. $21 \mathrm{~N}^{\circ}$ 5, pp. 525-536. 2010.

[3] P. Drucker. "La revolución educativa". En: Etzioni A. y Etzioni E. comp. 1968: "Los cambios sociales. Fuentes tipos y consecuencias". FCE. Ciudad de México, México. 1959.

[4] L. Hsiu-Fen. "Contextual factors affecting knowledge management diffusion in SMEs". Industrial Management \& Data Systems. Vol. $114 \mathrm{~N}^{\circ}$ 9, pp. 1415 - 1437. 2014. doi: 10.1108/IMDS-08-2014-0232

[5] G. Mora y E. Vidal. "La Economía de la Educación Superior". Roddy Begg eds.: "En el dialogo entre la enseñanza de la educación superior y la práctica". Kluwer Publicaciones. pp. 121-134. 2003.

[6] P. Drucker. "La Sociedad Postcapitalista". Grupo Editorial Norma. Bogotá, Colombia. 2004.
[7] S. Wilhelm y S. Gueldenberg. "Customer knowledge management: State of the art and future research directions". International Journal of Knowledge Management Studies. Vol. $53 \mathrm{~N}^{\circ}$ 4, pp. 265-384. 2014. doi: 10.1504/ IJKMS.2014.067220.

[8] M. García-Fernández. "Influence of quality management over innovation performance through knowledge management. A case study". Innovar. Vol. $26 \mathrm{~N}^{\mathrm{o}}$ 61, pp. 45-64. 2016. doi: 10.15446/innovar.v26n61.5711

[9] A. Honarpour, A Jusoh and K. Md Nor. Knowledge management, total quality management and innovation: A new look. Journal of Technology Management and Innovation. Vol. $7 \mathrm{~N}^{\mathrm{o}}$ 3, pp. 22-31. 2012. doi: 10.4067/S0718-27242012000300003.

[10] F. Díaz-Piraquive, V. Medina-García and R. González-Crespo. Knowledge management model for project management. En: Uden, L., Heričko, M., y I-Hsien, T. eds. Knowledge Management in Organizations. Vol. 1 Springer International Publishing. Berna, Suiza. pp. 235-247. 2015.

[11] K. Wing. Beginning a journey of knowledge management in a secondary school. Journal of Knowledge Management. Vol. $20 \mathrm{~N}^{\mathrm{o}}$ 2, pp. 364 - 385. 2016. doi: 10.1108/ JKM-04-2015-0155.

[12] V. Pekka-Economoul and S. Hadjidema. "Innovative organizational forms that add value to both organizations and community: The case of knowledge management". European Research Studies. Vol. 14 N $^{\mathrm{o}} 2$, pp. 81-96. 2011.

[13] M. Castells. "La era de la información. Economía, Sociedad y Cultura". La Sociedad Red. Vol. 1. Allianza Editorial. Madrid, España. 1996.

[14] P. Gottschalk. "Gestión del conocimiento en las empresas de servicios profesionales: estudio de las utilidades de las TI en los bufetes de abogados. Sistemas de Gestión del Conocimiento". Thomson Editores. Madrid, España. pp. 97-110. 2002.

[15] J. Ranjan and V. Bhatnagar. "Role of knowledge management and analytical CRM in business: Data mining based framework". The Learning Organization. Vol. $18 \mathrm{~N}^{\circ} 2$, pp. 131 - 148. 2011. doi: $10.1108 / 09696471111103731$ 
[16] C. E. Reginato and O.D. Gracioli. "Strategic management of information through the use of competitive intelligence and knowledge management - A study applied to the furniture industry in Rio Grande do Sul, Brazil". Gestao e Producao. Vol. 19 N $^{\circ}$ 4, pp. 705-716. 2012. doi: 10.1590/S0104-530X2012000400004.

[17] Sociedad del Conocimiento y Desarrollo Humano. "Fundación Iberoaméricana del Conocimiento". Fecha de consulta: 13 de junio de 2016. URL: http://www.ripei.org/ files/Sociedad $\% 20 \mathrm{del} \% 20$ conocimiento $\% 20$ y\%20desarrollo\%20humano.pdf

[18] I. Nonaka y H. Takeuchi. "La empresa creadora de conocimiento". En: "Gestión del Conocimiento". Deusto-Harvard Business Review. 2000.

[19] A. M. Ortegon-Alvarez, A. L. Lasso and A.V. Steil. "Organizational strategy and the knowledge management cycle: Bukowitz and william's model in practice". Espacios. pp. 377. 2016.

[20] L. Blas. "Gestión del Conocimiento, ¿De qué estamos hablando?" Revista Petrotecnia, Buenos Aires, Argentina. pp. 12-21. 2009.

[21] E. Bueno. "Enfoques principales y tendencias en dirección del conocimiento". En: Gestión del conocimiento: desarrollos teóricos y aplicaciones. Ediciones La Coria. Cáceres, España. 2002.

[22] P. Senge. "La quinta disciplina: el arte y la práctica de la organización abierta al aprendizaje". Granica. Buenos Aires, Argentina. 2005.

[23] A. Nazatul, M. Khairul, M. Dang, S. Arpah and S. Munira. "Knowledge management implementation in a government research institute in Selangor, Malaysia”. IBIMA Publishing. Selangor, Malaysia. 2013.

[24] M.S. Alkhanbashi. "Knowledge management challenges in the public sector". Knowledge Management. Vol. $15 \mathrm{~N}^{\circ}$ 4, pp. 23-42. 2015.

[25] J. Hoyos y A. Valencia. "El papel de las TIC en el entorno organizacional de las pymes". Trilogía. Ciencia, Tecnología y Sociedad. Vol. 7, pp 105-122. 2012.

[26] A. Valencia, G. González and M. Castañeda. "Structural equation model for studying the mobile-learning acceptance". IEEE Latin America Transactions. Vol 14. $\mathrm{N}^{\circ}$ 4, pp. 1988-1992, 2016. DOI: 10.1109/ TLA.2016.7483544
[27] L. chavarria, A. Valencia and J. Bermudez. "Agent-based Model for the Analysis of Technological Acceptance of Mobile Learning". IEEE Latin America Transactions. Vol. $15 \mathrm{~N}^{\circ}$ 6, pp. 1121-1127, 2017.

[28] A. Valencia, O. Salazar and D. Ovalle. "Improving the Entrepreneur's Market Research Strategies Learning Process Using the MaREMAS Environment". En: Highlights on Practical Applications of Agents and Multi-Agent Systems. Springer Berlin Heidelberg, pp. 363-374. 2013. DOI: 10.1007/978-3-642-38061-7_34

[29] J. Bermúdez, S. Chalela, J. Valencia and A. Valencia. "Research Trends in the Study of ICT Based Learning Communities: A Bibliometric Analysis". EURASIA Journal of Mathematics, Science \& Technology Education, Vol. $13 \mathrm{~N}^{\circ}$ 4, pp. 1539-1562, 2017.

[30] E. Villa Enciso, E. Picón, A. Valencia-Arias and C. Jiménez. "Analysis of University Management of Emerging Technologies and Recommendations for Developing Countries". Turkish Online Journal of Educational Technology-TOJET, Vol. 16 $\mathrm{N}^{\circ}$ 2, pp. 1-10. 2017

[31] G. Pérez. "Investigación cualitativa. Retos, interrogantes y métodos". La Muralla. España. 1994.

[32] O. G. León y I. Montero. "Métodos de investigación en Psicología y Educación". McGraw-Hill. Madrid. España. 2002.

[33] A. Kianto, M. Vanhala and P. Heilmann. "The impact of knowledge management on job satisfaction". Journal of Knowledge Management. Vol. $20 \mathrm{~N}^{\circ}$ 4, pp. $621-636$. 2016. doi: http://dx.doi.org/10.1108/ JKM-10-2015-0398.

[34] C. Bernal. Aproximación a la medición de la gestión del conocimiento empresarial. Fecha de consulta: 7 de octubre de 2016. URL: http://publicaciones.eafit.edu.co/ index.php/administer/article/view/183/198\#. UXrNp6JmiSo.

[35] M.E. Zaei and P Kapil. "The role of intellectual capital in promoting knowledge management initiatives". Knowledge Management and E-Learning. Vol. $8 \mathrm{~N}^{\mathrm{o}}$ 2, pp. 317-333. 2016.

[36] J. Hartley. "Case studies in organizational research". En: Catherine Cassell \& Gillian 
Symon Eds., "Qualitative methods in organizational research, a practical guide". Sage. Londres, Reino Unido. pp. 208-229. 1994.

[37] C. Ling-hsing and L. Tung-Ching. "The role of organizational culture in the knowledge management process". Journal of Knowledge Management. Vol. $19 \mathrm{~N}^{\circ} 3$, pp. $433-455$. 2015. doi 10.1108/JKM-08-2014-0353.

[38] H., Vazifedost and B. Nemati. "Factors affecting knowledge management in the ministry of communications and information technology". International Business Management. Vol. $10 \mathrm{~N}^{\circ}$ 10, pp. 1784-1792. 2016. doi: 10.3923/ibm.2016.1784.1792.

[39] M. Vidal y A. Araña. "Gestión de la información y el conocimiento". Educación Médica Superior, Vol. 26 Nº 3, pp. 474-484, 2012.

[40] M. Monagas-Docasal. "El capital intelectual y la gestión del conocimiento". Ingeniería Industrial, Vol. $33 \mathrm{~N}^{\mathrm{o}}$ 2, pp. 142-150, 2012

[41] J. Acosta y A. Fischer. "Condiciones de la gestión del conocimiento, capacidad de innovación y resultados empresariales. Un modelo explicativo". Pensamiento \& Gestión. Vol. 35, pp. 25-63. 2012

[42] F. Cárcel y C. Roldan. "Principios básicos de la Gestión del Conocimiento y su aplicación a la empresa industrial en sus actividades tácticas de mantenimiento y explotación operativa: Un estudio cualitativo". Intangible capital, Vol. $9 \mathrm{~N}^{\circ}$ 1, pp. 91-125, 2013. URL: http://hdl.handle.net/2099/13235

[43] G. Arboleda. "Percepción que los gerentes tienen acerca de la gestión del conocimiento en las instituciones de salud, Valle de Aburrá, 2011. Facultad Nacional de Salud Pública, Vol. 32 N². 2014.

[44] A. Romo, M. Villalobos y L. Guadalupe. "Gestión del conocimiento: estrategia para la formación de investigadores". Sinéctica. Vol. 38, pp. 1-20, 2012.

[45] P. Drucker. "La Sociedad Postcapitalista". Grupo Editorial Norma. Bogotá, Colombia. 2004.

[46] L. Giraldo y D. Montoya. "Aplicación de la metodología Commonkads en la Gestión del Conocimiento". Revista CEA. Vol. $1 \mathrm{~N}^{\circ} 2$, pp. 99-108. 2015
[47] V. Rodríguez-Lora, M. Henao-Cálad and A. Valencia Arias. "Taxonomies of techniques and tools for Knowledge Engineering: guide for knowledge project development". Ingeniare. Revista chilena de ingeniería. Vol. $24 \mathrm{~N}^{\mathrm{o}} 2$, pp 351-360. 2016. DOI: 10.4067/ S0718-33052016000200016

[48] R. Villafuerte-Godínez y J. Leiva. "Cómo surge y se vincula el conocimiento relacionado con el desempeño en las Pymes: un análisis cualitativo". Revista CEA, Vol. $1 \mathrm{~N}^{\mathrm{o}} 2$, pp. 37-48. 2015. 